\title{
A new wake model and comparison of eight algorithms for layout optimization of wind farms in complex terrain
}

\author{
Brogna, Roberto; Feng, Ju; Sørensen, Jens Nørkær; Shen, Wen Zhong; Porté-Agel, Fernando
}

Published in:

Applied Energy

Link to article, DOI:

10.1016/j.apenergy.2019.114189

Publication date:

2020

Document Version

Early version, also known as pre-print

Link back to DTU Orbit

Citation (APA):

Brogna, R., Feng, J., Sørensen, J. N., Shen, W. Z., \& Porté-Agel, F. (2020). A new wake model and comparison of eight algorithms for layout optimization of wind farms in complex terrain. Applied Energy, 259, [114189]. https://doi.org/10.1016/j.apenergy.2019.114189

\section{General rights}

Copyright and moral rights for the publications made accessible in the public portal are retained by the authors and/or other copyright owners and it is a condition of accessing publications that users recognise and abide by the legal requirements associated with these rights.

- Users may download and print one copy of any publication from the public portal for the purpose of private study or research.

- You may not further distribute the material or use it for any profit-making activity or commercial gain

- You may freely distribute the URL identifying the publication in the public portal 


\title{
A new wake model and comparison of eight algorithms for layout optimization of wind farms in complex terrain
}

\author{
Roberto Brogna ${ }^{\mathrm{a}}$, Ju Feng ${ }^{\mathrm{b}, *}$, Jens Nørkær Sørensen ${ }^{\mathrm{b}}$, Wen Zhong Shen ${ }^{\mathrm{b}}$, \\ Fernando Porté-Agel ${ }^{a}$ \\ ${ }^{a}$ Wind Engineering and Renewable Energy Laboratory, École Polytechnique Fédérale de \\ Lausanne (EPFL), CH-1015 Lausanne, Switzerland \\ ${ }^{b}$ Fluid Mechanics Section, Department of Wind Energy, Technical University of Denmark, \\ DK-2800 Kgs. Lyngby, Denmark
}

\begin{abstract}
Layout optimization of wind farms constitutes an important and challenging task in complex terrain. This is especially due to the complex interactions of the boundary layer flows in complex terrain and wind turbine wakes, which renders wake modelling in complex terrain difficult. This study tackles this challenge with a new engineering wake model, which is developed by superposing a Gaussian shape wake model on top of the background flow field, assuming that the centerlines of wind turbine wakes follow the streamlines of the background flow field. The model is found to predict wind turbine wakes in complex terrain with good accuracy and at the same time it is computationally cheap to run for optimization applications. Comparisons with high fidelity simulations and field measurements for a real wind farm with 25 turbines in complex terrain demonstrate its effectiveness. A systematic comparison of eight optimization algorithms, which includes two gradient-based and six gradient-free algorithms, is also carried out for the layout optimization problem in complex terrain. To accelerate the optimization process, a double-stage approach is proposed, which optimizes the objective function first neglecting wake effects and then, in the second stage, including them. While all the tested optimization algorithms can improve the original wind farm layout, random search, local search, and pattern search are found to be the top three algorithms in terms of optimization results and computational cost.
\end{abstract}

Keywords: Wind farm design, complex terrain, wake model, streamlines, layout optimization, systematic comparison

\footnotetext{
${ }^{*}$ Corresponding author

Email address: jufen@dtu.dk (Ju Feng)
} 


\section{Introduction}

During the last two decades, wind power has become a mature and important player in the global energy marketplace. The latest statistics published by the Global Wind Energy Council (GWEC) shows that the global cumulative installed wind capacity increased from $24 \mathrm{GW}$ to $591 \mathrm{GW}$ in the period from 2001 to 2018 , which represents an impressive average growth of $20.7 \%$ annually [1].

Together with the rapid growth of installed wind capacity, wind farms proliferate worldwide. A task of central importance to the success of a wind farm is choosing its initial layout or design. This is because a wind farm design, once chosen in the development stage and applied in the construction and installation stage, will have large and long-lasting effects on the operation stage of the project, which typically spans from 20 to 25 years. Yet, once constructed and installed, it becomes nearly impossible or prohibitively expensive to change the wind farm design.

A complete wind farm design includes the design tasks of many components and/or sub-systems, such as type, number and locations of wind turbines, turbine foundations, electrical systems, and access road network (for onshore wind farms). Among these different design tasks, determining wind turbine locations, usually referred to as layout optimization or micro-siting, is crucial. Optimizing a wind farm's layout concerns determining the optimal locations of wind turbines with regards to a single/multiple objective(s), while satisfying certain constraints and requirements [2].

The seminal work in the field of wind farm layout optimization was done by Mosetti et al. in 1994 [3], in which the layout optimization problem to minimize the cost of energy under idealized wind conditions was solved using a Genetic Algorithm (GA). Following this work, a large number of studies have investigated this problem, with the majority focusing on wind farms located on flat terrain or offshore 4 .

Different formulations of the layout optimization problem have been tried in different studies. For design variables, most of the studies considered only locations of a given number of turbines [4, while few studies considered also other elements. For example, Chen et al. [5] considered hub heights and locations of turbines simultaneously; Vasel-Be-Hagh and Archer [6] optimized the hub heights alone while assuming a fixed number and locations of turbines; and Feng and Shen [7] included number, types and locations of turbines as design variables. For objective functions, popular choices include power/energy production [8, 9] and (levelized) cost of energy [7, 10]. Other objective functions have also been used, for example, González et al. [11 investigated the maximization of net present value (NPV), Mayo and Daoud [12] considered energy

efficiency and the degree of visual impact simultaneously, and robustness of power production under changing wind conditions was considered in [13. Common constraints considered in the layout optimization studies include the wind farm boundary and minimal distance requirements [4.

Various optimization algorithms have been applied to solve the layout opti- 
mization problem, among which GA is the most widely used, e.g., in [10, 11]. More advanced version of GA has also been proposed for solving this problem, such as the self-informed GA with information guided exploitation in [14]. Other algorithms have also been tried, such as extended pattern search 15, particle swarm optimization (PSO) [16], greedy search [6, 17, local search [18] and random search [7, 19. Nevertheless, systematic comparative studies between different algorithms are still largely missing in the literature.

Among the published studies on wind farm layout optimization, very few considered wind farms in complex terrain as the majority of them assumed wind farms on flat terrain or at offshore sites 4 . This can be partially explained by the difficulty of modelling wind turbine wakes in complex terrain 20. As Castellani et al. 21 found by using numerical and field measurement data, the performance of wind farms in complex terrain is influenced by a complex interplay of terrain-driven flow and wake flow of wind turbines. Similar complexity and the importance of considering terrain effects were also observed in a recent study 22] for a wind farm in complex terrain based on LiDAR (Light Detection and Ranging) measurements and SCADA (Supervisory Control and Data Acquisition) data.

In the general field of wind farm layout optimization, the most widely used wake model is the Jensen wake model [23], as shown in the review work by Shakoor et al. 24. Other engineering wake models have also been developed for various wind energy applications 25. For example, Bastankhah and PortéAgel [26] proposed a Gaussian shape wake model, which has been applied for offshore wind farm layout optimization in 27; ; a similar model has also been developed by Gao et al. [10]; Sun and Yang [28] developed an analytical threedimensional wind turbine wake model which accounts for the wind variation in the vertical direction; and more recently Cheng et al. 29] developed a new analytical wake model based on the Monin-Obukhov similarity theory. Nevertheless, most engineering wake models, such as the aforementioned ones, only work for wind farms on flat terrain or at offshore sites. More recently, Ti et al. [30] proposed an innovative way to model wind turbine wakes using machine learning. In this study, a high fidelity data set of wake flow after a turbine was first obtained by RANS (Reynolds-averaged Navier-Stokes equations) simulation with a turbine modelled by an actuator disk model with rotation. Then an artificial neural network (ANN) model was trained using this data set to estimate the wind speed and turbulence intensity of the wake flow. The validation of this ANN model reveals promising results for an offshore wind farm, but its applicability in complex terrain needs to be further investigated.

Among the few studies that considered wind farm design in complex terrain, Song et al. [31, 32] relied on a wake model based on virtual particle simulation using either a bionic method or a greedy algorithm [31. An adapted Jensen wake model was proposed by Feng and Shen for wind farms in complex terrain and applied in the layout optimization of a wind farm on a 2D Gaussian hill 33. This wake model was later adopted in a design optimization framework that can consider real wind farms in complex terrain and tested on a wind farm with 25 turbines at a real complex terrain site [34. Kuo et al. 35] 
solved the layout optimization problem for wind farms in complex terrain by coupling computational fluid dynamics (CFD) with mixed-integer programming (MIP), in which the wake effects were approximated by CFD simulations iteratively. More recently, a new analytical modelling framework for wind turbine wakes over two-dimensional hills was developed by Shamsoddin and Porté-Agel [36, in which the modelling process consists of two steps: the first step deals with the effect of the pressure gradient on the wake evolution, and the second step considers the effect of the hill-induced streamline distortion. While this model achieved good agreement with large-eddy simulation (LES) results, the assumption of two-dimensional hills limits its applicability for wind farms in real complex terrain sites.

In this study a new engineering wake model is proposed, which can work for wind farms in real three-dimensional (3D) complex terrain sites. The model is developed by superposing a Gaussian shape wake model on top of the CFD obtained background flow field, assuming the centerlines of wind turbine wakes follow the streamlines in the background flow field. The model is validated for the layout of a wind farm with 25 turbines at a real complex terrain site. The analysis shows that this model can predict the wake flow of wind farms in complex terrain with good accuracy in a short time, thus making it very suitable for applications in layout optimization.

In view of the lack of systematic comparative studies of optimization algorithms, we also compare the performance of eight algorithms for the layout optimization problem of wind farms in complex terrain. These algorithms include two gradient-based and six gradient-free algorithms, among which the best-performing ones for this problem are identified. A double-stage optimization approach is also proposed to further accelerate the optimization process for wind farm design in complex terrain.

\section{Studied wind farm}

To demonstrate the wake model proposed in this study and its application in layout optimization, a real wind farm is chosen and studied. This wind farm, which is located at a complex terrain site in Northwest China, is composed of 25 wind turbines. These turbines are of the same type and their technical specifications are summarized in Table 1. Performance characteristics of this type of turbine are shown in Fig. 1. 
Table 1: Specifications of wind turbines in the studied wind farm.

\begin{tabular}{cc}
\hline Variable & Value \\
\hline Rotor diameter & $93 \mathrm{~m}$ \\
Rated power & $2.0 \mathrm{MW}$ \\
Hub height & $67 \mathrm{~m}$ \\
Cut-in wind speed & $3 \mathrm{~m} / \mathrm{s}$ \\
Rated wind speed & $12 \mathrm{~m} / \mathrm{s}$ \\
Cut-out wind speed & $25 \mathrm{~m} / \mathrm{s}$ \\
Power control & Variable speed, pitch regulated \\
\hline
\end{tabular}

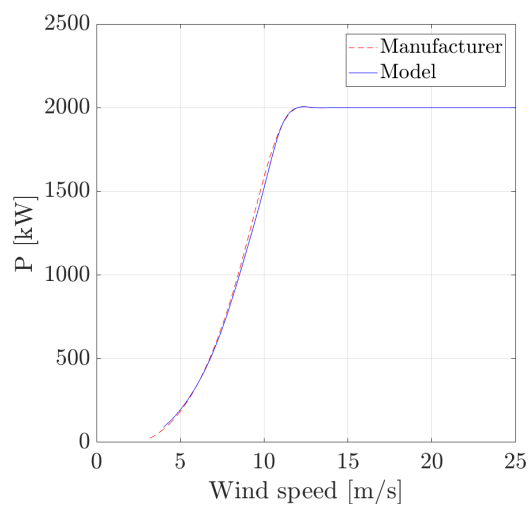

(a)

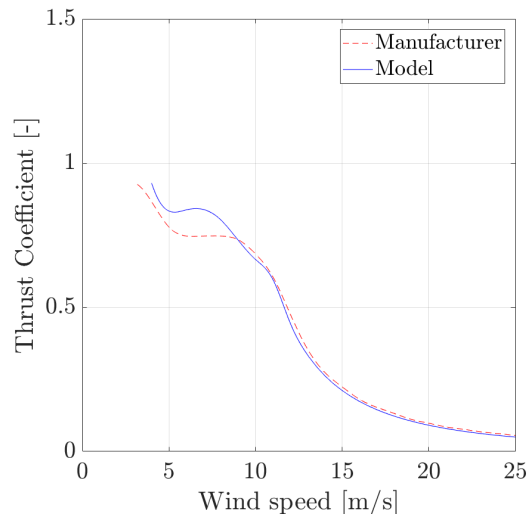

(b)

Figure 1: Power curve (a) and thrust coefficient curve (b) of the turbine in the studied wind farm.

The topography of the terrain and the elevation contour within a rectangle domain of size $6 \mathrm{~km}$ by $4 \mathrm{~km}$, along with the positions of the turbines and two met masts, are shown in Fig. 2 . 


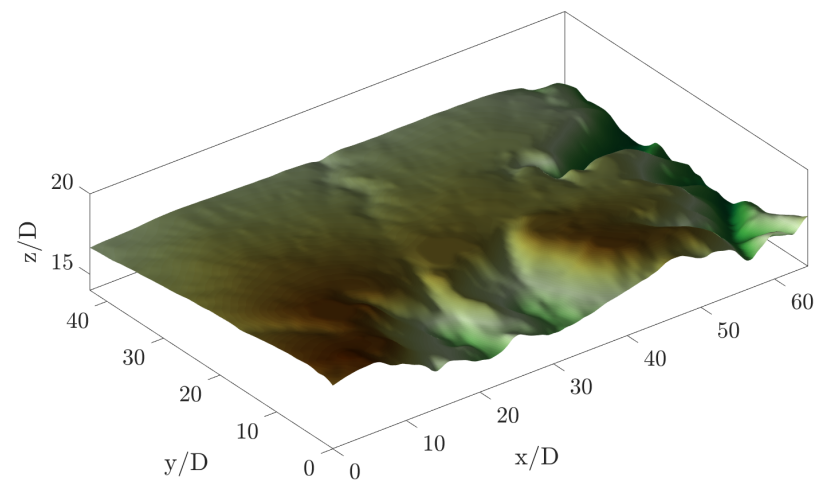

(a) Terrain topography

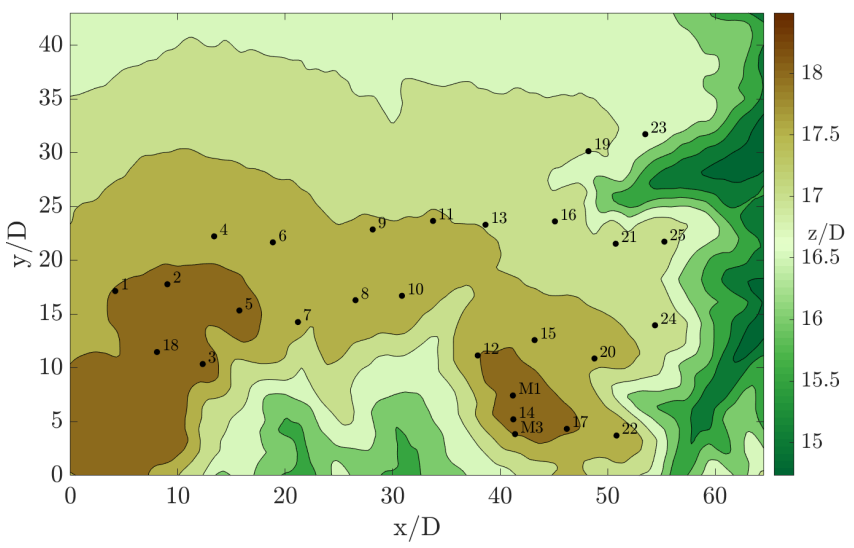

(b) elevation contour

Figure 2: Terrain topography (a) and elevation contour (b) of the studied wind farm. The positions of twenty-five turbines are shown with numbers along with the two met masts M1 and M3.

Note that the two met masts have a height of $70 \mathrm{~m}$ and that the coordinates shown in Fig. 2 are the scaled and shifted version of the original coordinates. The scaling factor is equal to the rotor diameter $D$, i.e., $93 \mathrm{~m}$, and the shifting distances are chosen so that the origin of the $x-y$ plane lies at the southwest corner of the domain.

\section{Wake modelling}

For wind farms on flat terrain or at offshore sites, wake modelling is a relatively easy task as there are well-established methods using engineering wake models [37, such as the Jensen wake model [23], the Frandsen wake model [38, or the more recently developed Gaussian wake model (GWM) [26]. However, these wake models were all developed in assuming flat terrain or offshore site. Due to the interaction between wake flow and terrain perturbation effects, wake 
modelling is much more difficult for wind farms in complex terrain [20]. Thus these wake models can't be directly applied for wind farms in complex terrain. While this challenge can be tackled by using CFD with a reasonable accuracy 39, 40, the high computational costs make CFD methods generally unsuitable for wind farm design optimization.

As most optimization algorithms used in wind farm design require evaluating a large number of design candidates, a fast wake model is required to estimate the wake flows at each turbine's location with reasonable accuracy in a short time.

The adapted Jensen wake model proposed by Feng and Shen [33] in 2014 is one such example. In this model, the centerline of the wake generated by a wind turbine is assumed to follow the terrain at the same hub height level above the ground and go along with the local wind direction. The same assumptions on wake deficit profile and its expansion are made as in the original Jensen wake model [23. The schematic of this model is shown in Fig. 3.

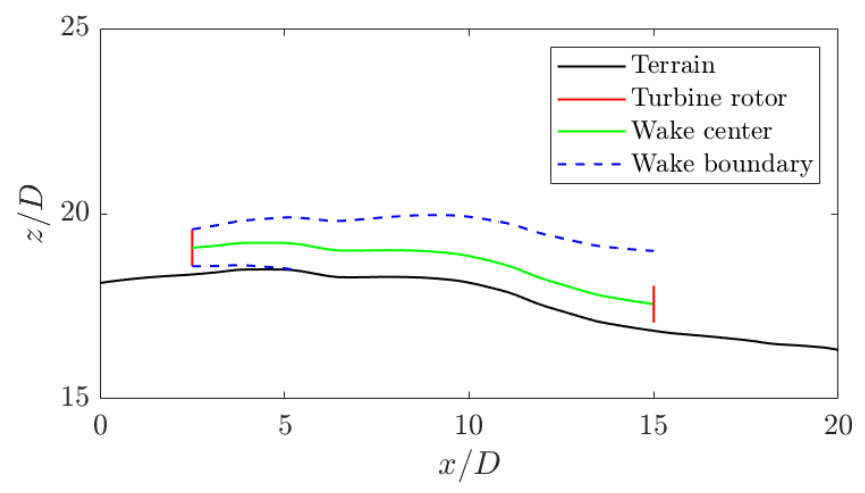

Figure 3: Schematic of the adapted Jensen wake model [34.

This model was first used in an idealized layout optimization problem, i.e., a wind farm with 25 turbines on a Gaussian 2D hill [33. Recently, it was also employed in a more realistic optimization framework and tested on the design optimization of a real wind farm in complex terrain [34, which is, in fact, the same wind farm as described in Section 2, Random search algorithm, a metaheuristic algorithm developed specifically for wind farm layout optimization in [19], was used in this framework.

While fast to compute and able to obtain reasonable results, the adapted Jensen wake model suffers from two main limitations: (1) the wake centerline is assumed to follow the terrain along with the local wind direction, (2) the wake deficit profile is assumed to be uniform inside the wake boundary, i.e., a top-hat shape is implicitly assumed. These two assumptions do not match with the physical reality. First, due to the terrain's perturbation effects on the flow filed, a wake flow in complex terrain does not go directly along the inflow wind at the rotor. Second, the deficit profile of a fully developed wake flow actually takes a Gaussian shape. This has been shown by Schlichting in his classic textbook 
'Boundary Layer Theory' [41, in which he derived an analytical solution for the wakes of blunt bodies. The same phenomenon has also been observed for wind turbine wakes, both in wind tunnel experiments 42 and field measurement [4, 44.

To overcome these limitations and better capture the physical reality, a new wake model for wind turbines in complex terrain is proposed. This new model takes the same basic approach as the adapted Jensen wake model, i.e., superposing an analytical engineering wake model, which works for wind turbines on flat terrain or at offshore sites, on top of a background flow field. Note that a background flow field refers to the flow field over a terrain without any turbines.

In contrast to the adapted Jensen model, the new model makes several assumptions that agree better with the physical reality. These are listed as follows:

- Assumption 1: The centerline of a turbine's wake follows the streamline starting from the rotor centre in the background flow field.

- Assumption 2: In the background flow field, streamlines from positions at the hub height level above the ground can be approximated by streamlines from the same positions on the iso-surface at the same height, plus corrections accounting for the out of iso-surface displacements.

- Assumption 3: The wake deficit profile of an individual turbine takes a Gaussian shape.

To better illustrate these assumptions, a schematic diagram showing the wake of a wind turbine located on a complex terrain site is presented in Fig. 4. Note that in this figure, the streamline obtained by the model in Assumption 2 is denoted as pseudo 3D streamline, while the wake boundary is assumed to be the boundary corresponding to three standard deviations away from the wake centerline in the Gaussian wake profile. For comparison, the wake centerline used in the adapted Jensen wake model [33] is also shown in this figure as the terrain following curve. 


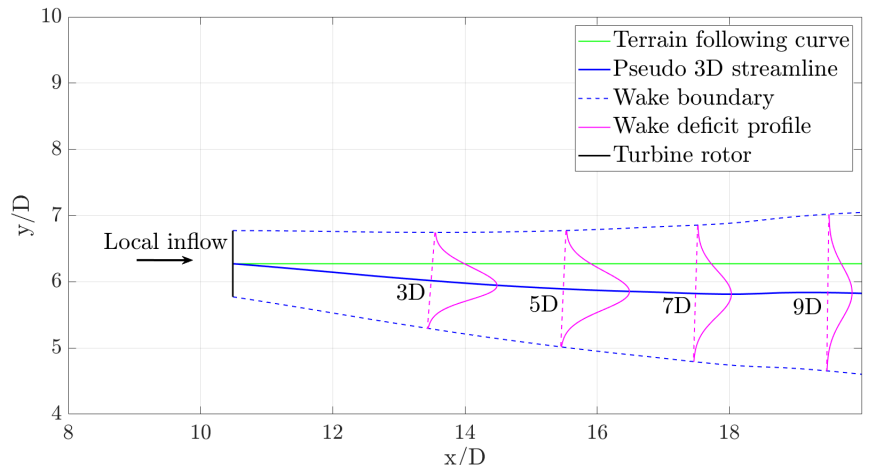

(a) Top view

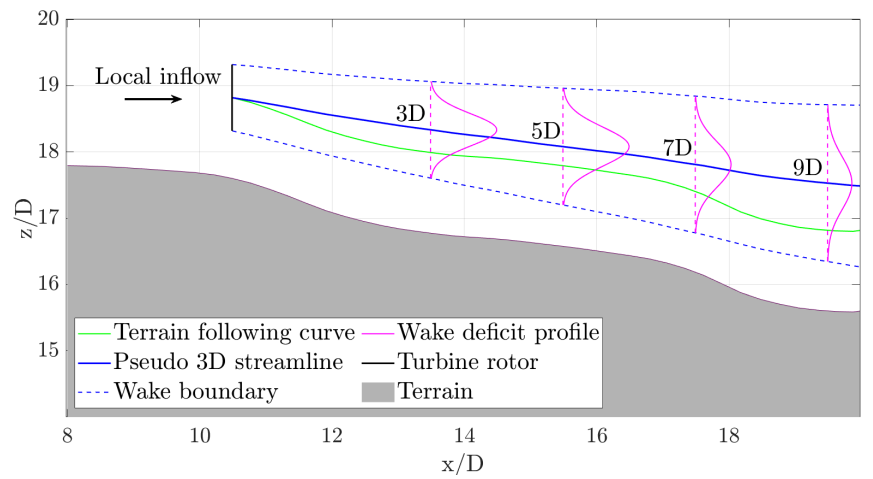

(b) Side view

Figure 4: Schematic diagram of wake flow of a wind turbine on a complex terrain site under the three assumptions for the proposed new wake model.

For a better understanding of the differences between the adapted Jensen wake model 33 and the proposed new wake model, 3D visualizations of these two models are also shown in Fig. 5 


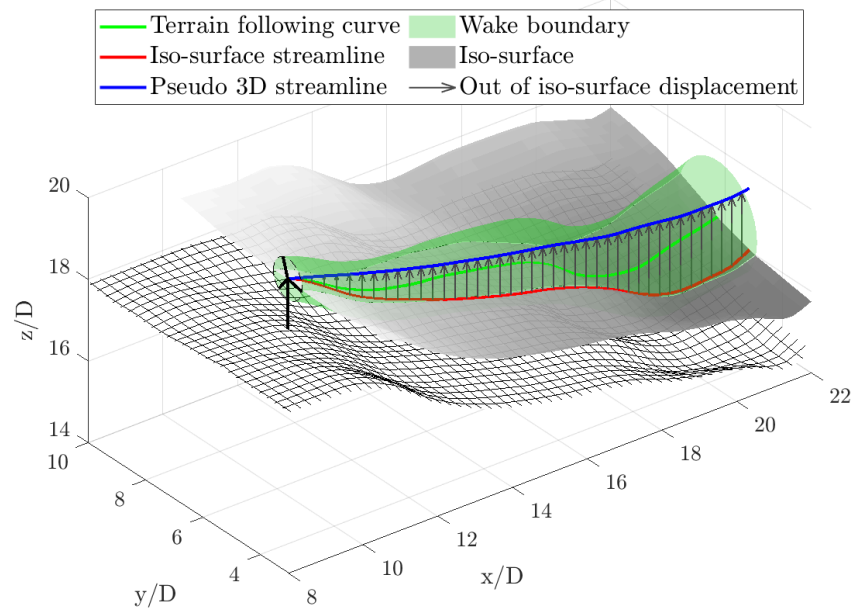

(a) Adapted Jensen wake model

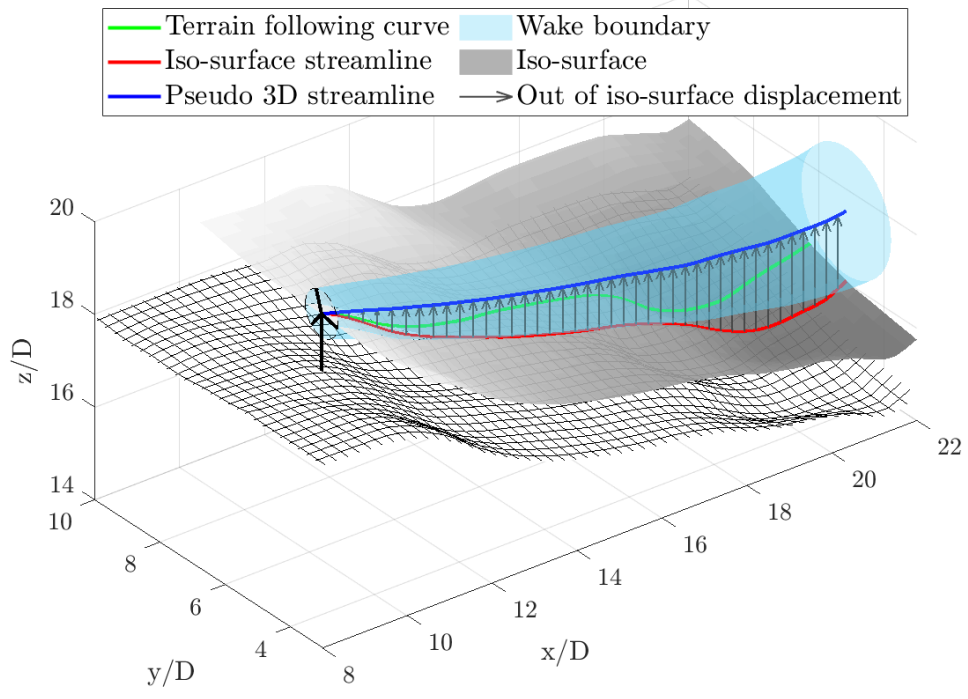

(b) Proposed new wake model

Figure 5: Comparison of the adapted Jensen wake model 33 and the proposed new wake model for a wind turbine at a complex terrain site.

From Figs. 4 and 5 , we could clearly see how the pseudo 3D streamline is obtained following Assumption 2 and the new model captures the threedimensional nature of the wake trajectory in complex terrain by making the wake centerline follows the pseudo 3D streamline. Besides, the more realistic Gaussian profile of the wake deficit is also considered. The detailed modelling method of this new model is presented in the following sub-sections. 


\subsection{Background flow field}

For complex terrain sites, the background flow field is affected by the terrain effects. Thus, streamlines in the background flow field exhibit a threedimensional nature, and calculating these streamlines will require some information on the three-dimensional flow field. It is thus unfavourable to calculate these streamlines during the design optimization process, since this will slow down the computation and jeopardize the applicability of the model.

To limit the computational burden while capturing the three-dimensional nature of real streamlines in the background flow field, Assumption 2 as summarized above, is used. Based on this assumption, only one layer of velocity field information needs to be stored and used, i.e., those velocity components on the iso-surface at the hub-height level above the ground. This reduces the required data storage and enables to pre-compute and store the information of a group of streamlines for each inflow wind direction sector.

Without loss of generality, the background flow field can be modelled by using some CFD tools for a number of inflow wind direction sectors. Then for each sector, the CFD results can be parsed to extract the wind velocity components along the directions $x, y, z$ for the iso-surface at the hub-height level above the ground, which are denoted as $\left(u_{x}, u_{y}, u_{z}\right)$. These velocity components can then be stored on a rectangular grid covering the wind farm area of interest.

In this study, the background flow field is modeled by WAsP CFD [45] for 12 sectors, and velocity components on the iso-surface at a height of $67 \mathrm{~m}$ above the terrain is stored on a grid over a rectangular domain of size $6 \mathrm{~km}$ by $4 \mathrm{~km}$. This grid is composed of cells with size of $25 \mathrm{~m}$ by $25 \mathrm{~m}$. This chosen grid size is based on the resolution of the map file and the mean horizontal size of the computational grid used in WAsP CFD. For any points not located on the nodes of the grid, linear interpolation is used to find the related velocity components. Comparing to the rotor diameter of turbine $(93 \mathrm{~m})$, the resolution of the grid ( $25 \mathrm{~m}$ by $25 \mathrm{~m}$ ) is sufficient in the present case.

As an example, the wind velocity components along the $x$ and $y$ directions, normalized to the far field inflow wind speed $U_{\infty}$, on the the iso-surface at the hub-height level above the ground under a given inflow sector is shown in Fig. 6. From this figure, we can clearly see the distortion of the background flow field caused by terrain effects. When modelling the wake effects, the influence of terrain effects on different turbines' local inflow conditions can therefore be well captured by the CFD obtained background flow field results. 


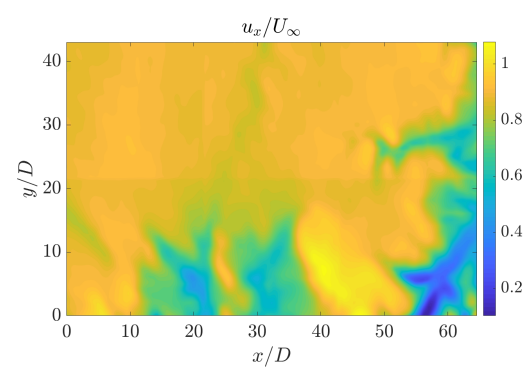

(a)

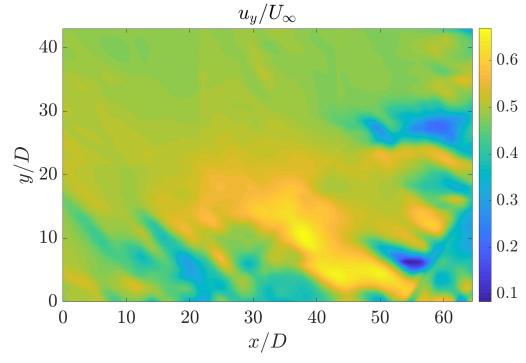

(b)

Figure 6: Contour of normalized velocity components at $67 \mathrm{~m}$ height level along the $x$ and $y$ directions for the inflow wind direction of $240^{\circ}$ for the studied wind farm.

For the purpose of this study the flow field on the iso-surface is expressed in terms of three components: one normal to the iso-surface $\left(u_{n}\right)$ and two parallel to the iso-surface and perpendicular to each other, with one lying in the $x-z$ plane $\left(u_{\tau, x}\right)$ and one in the $y-z$ plane $\left(u_{\tau, y}\right)$, respectively. This resulting expression representing the iso-contour can be obtained by projecting the velocity vectors defined by velocity components $\left(u_{x}, u_{y}, u_{z}\right)$ onto the iso-surface,

$$
\vec{u}=\left(u_{\tau, x}, u_{\tau, y}, u_{n}\right)
$$

Following Assumption 2, streamlines lying on the iso-surface are computed based on the velocity components $\vec{u}_{\tau}=\left(u_{\tau, x}, u_{\tau, y}\right)$.

Being subjected to a normal component, streamlines will not remain on the iso-surface, but they will deviate above or below the aforementioned curved iso-surface. As a consequence, it is necessary to compute this perpendicular deviation by considering at each point the normal component of the velocity field.

For the infinitesimal displacement along the curve $d s$, the streamline will deviate out of the plane by the amount:

$$
d \vec{n}=\frac{\left|u_{n}\right|}{\left\|\vec{u}_{\tau}\right\|} d s \cdot \hat{n}
$$

The total deviation perpendicular to the iso-surface at a certain point is obtained by integration along the curve. This approach is demonstrated in Fig. 7. where a streamline lying on the iso-surface is compared to the streamline that deviates from the iso-surface. 


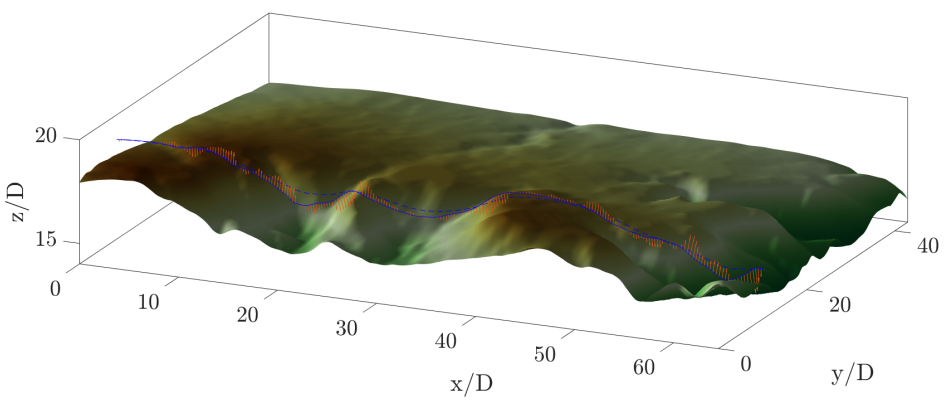

(a)

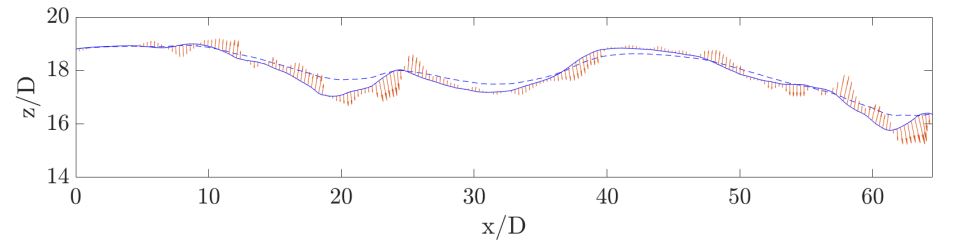

(b)

Figure 7: Streamline deviation out of the iso-surface plane for a wind from $270^{\circ}$. The original streamline, lying on the iso-surface is displayed (solid line) along with the new streamline after the deviation out of the iso-surface is taken into account (dotted line). The normal velocity component acting on the streamline is represented as well.

As shown in Fig. 7, adding the correction to account the deviation out of the iso-surface, tends to yield smoother streamlines with respect to those lying on the iso-surface.

To enable faster calculations of wake effects between any two turbines, we need to pre-compute a group of streamlines for each inflow wind direction sector. In order to get a uniform distribution over the entire domain, each group of streamlines is a set of equally spaced lines at the starting points along the boundaries of the domain. As an example, the streamlines obtained for an inflow wind direction of $300^{\circ}$ is displayed in Fig. 8 . 


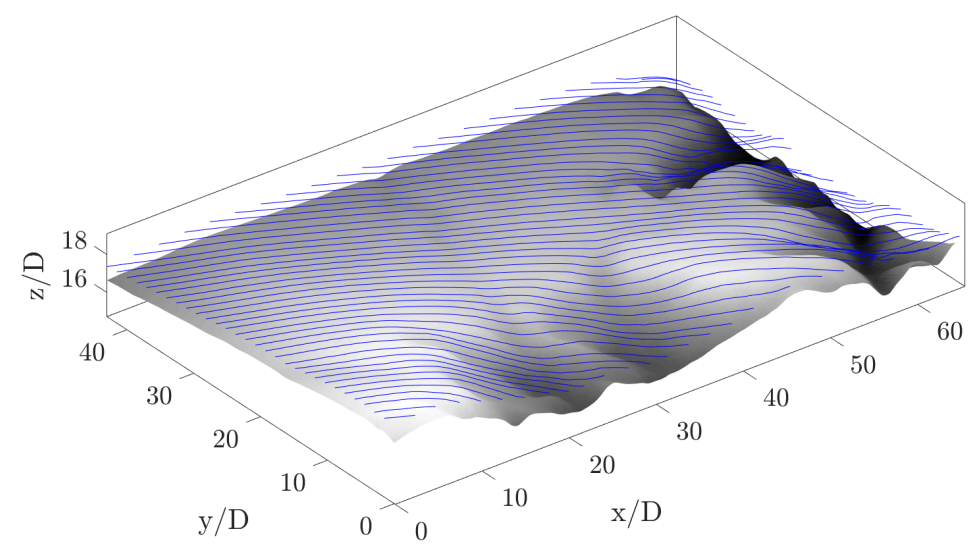

Figure 8: Streamlines over the terrain of the studied wind farm for the inflow wind direction of $300^{\circ}$.

\subsection{Engineering wake model}

The wind flow over a terrain is significantly affected by the presence of wind turbines, and, as a consequence, attention has been paid to the choice of the wake model to be used, to the trajectory followed by the wake, and to the interaction of the wake with other wind turbine wakes.

As discussed before, there are several widely used engineering wake models, including the Jensen wake model [23, the Frandsen wake model [38, and the Gaussian wake model (GWM) 26. Among these three models, the Jensen wake model and the Frandsen wake model both assume a simple top-hat shape wake deficit profile. This assumption contradicts with the Gaussian shape wake deficit profile predicted by theoretical derivation [41] and observed in wind tunnel experiments [42] and field measurement [43, 44] for wind turbine wakes. In GWM, the wake deficit profile is assumed to be a Gaussian shape, which agrees better with observations. In their paper presenting the GWM, Bastankhah and Porté-Agel [26] also showed that GWM has a better agreement with Large Eddy Simulation (LES) results, wind tunnel experiments and field measurements as compared to the Jensen wake model.

Based on the above considerations, GWM is selected in this study. The new engineering wake model is a modified version of the original GWM for applications in complex terrain. For a position downstream of a wind turbine of rotor diameter $D$ subject to a local inflow wind speed $U_{\infty}^{\text {loc }}$, the wake deficit governed by this new wake model is written as:

$$
\frac{\Delta U}{U_{\infty}^{l o c}}=\left(1-\sqrt{1-\frac{C_{T}\left(U_{\infty}^{\text {loc }}\right)}{8\left(k^{*} s / D+\epsilon\right)^{2}}}\right) \cdot \exp \left(-\frac{(r / D)^{2}}{2\left(k^{*} s / D+\epsilon\right)^{2}}\right)
$$

where $s$ denotes the downwind distance from the rotor to the position following the wake trajectory, $C_{T}\left(U_{\infty}^{\text {loc }}\right)$ is the thrust coefficient of the wind turbine at the 
inflow wind speed $U_{\infty}^{\text {loc }}, r$ represents the radial distance from the wake centerline from the rotor centre, $k^{*}=\partial \sigma / \partial x$ denotes the growth rate of the wake (assumed to be $k^{*}=0.042$ in this study), and $\epsilon$ is a parameter that depends on $C_{T}\left(U_{\infty}^{l o c}\right)$, given as:

$$
\epsilon=0.2 \sqrt{\frac{1}{2} \frac{1+\sqrt{1-C_{T}}}{\sqrt{1-C_{T}}}}
$$

Note that in the original version of GWM, which was developed for flat terrain, $s$ is the straight line distance along the inflow wind direction, i.e., along $x$, which can easily be calculated from the coordinates of the upwind turbine site and the downwind position. Thus, this new wake model can be viewed as a generalized version of the original GWM to cover different types of terrain.

Using the new wake model described by Eqs. (3) and (4), the velocity coutour at hub height level behind a wind turbine in flat terrain is shown in Fig. 9. Note that for flow field in flat terrain, the local inflow wind speed $U_{\infty}^{\text {loc }}$ is the same as the far field inflow wind speed $U_{\infty}$.

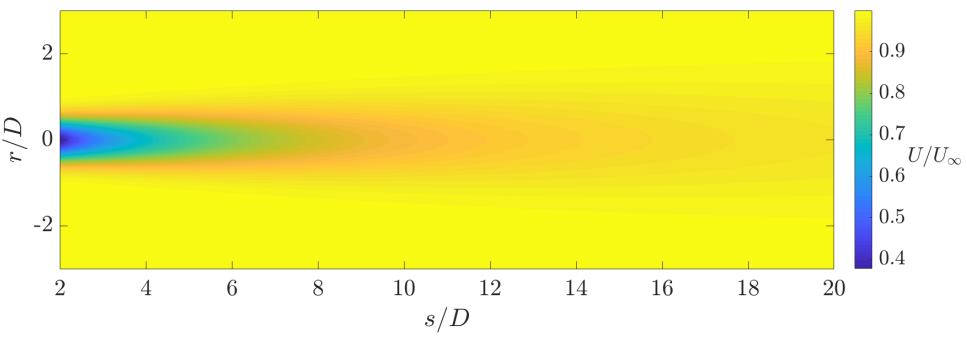

Figure 9: Velocity contour of the wake region at hub height level under inflow wind speed of $7 \mathrm{~m} / \mathrm{s}\left(C_{t}=0.747\right)$ according to the Gaussian wake model (top view).

For a wind turbine in complex terrain, the downwind distance $s$ needs to be computed along the three-dimensional streamline in the background flow field, which is much more complex than in the case of a flat terrain.

When the wake of an upwind turbine reaches a downwind turbine, the velocity deficit varies in the different points of the area spanned by the rotor. As a consequence, it is necessary to determine the average velocity deficit by integration over the rotor area $A$ :

$$
\frac{\Delta U_{\text {avg }}}{U_{\infty}^{\text {loc }}}=\frac{1}{A} \int_{A} \frac{\Delta U}{U_{\infty}^{\text {loc }}} d A
$$

\subsection{Superposition strategy}

To superpose the engineering wake model (GWM) onto the background flow field, we need to compute the downwind distance $s$ and radial distance $r$ from the upwind turbine rotor centre to any point in the rotor area of the downwind turbine, as seen in Eq. (3).

One strategy to tackle this task is to compute the three-dimensional streamline from the upwind turbine based on the full flow field information. However, 
as discussed in sub-section 3.1, this strategy is computationally too heavy for optimization applications. Thus, instead, a strategy to simplify the computations and lower the computational cost is used. This strategy requires first to pre-compute groups of streamlines using the approximation method summarized in Assumption 2 for all inflow wind direction sectors, and then to determine the distance between any two turbines based on these pre-computed and stored streamlines for any inflow wind direction sector.

Since the number of streamlines pre-computed and stored is limited, the following expedient is used to have a set of streamlines that passes exactly through each rotor. Each turbine is associated with the closest streamline and it is assumed that the streamline passing through the rotor would be parallel to it. As a consequence, it is sufficient to translate the streamline selected by an amount $\vec{\delta}=\left(\delta_{x}, \delta_{y}, \delta_{z}\right)$ to obtain a new one that crosses the rotor center. An example of this is displayed in Fig. 10.

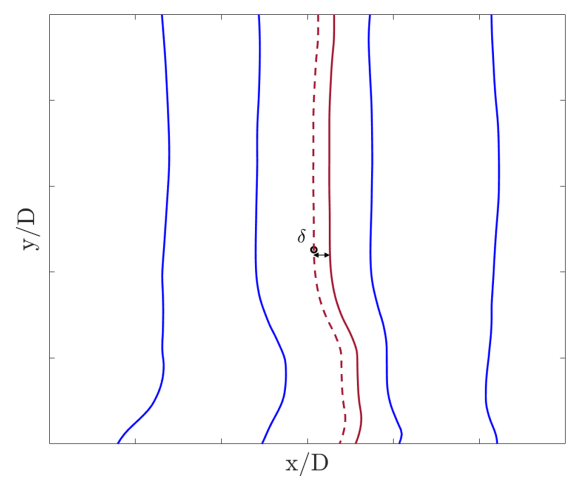

Figure 10: Streamline through the rotor (dash red) is assumed to be parallel to the closest streamline (red) (top view).

By using GWM, which assumes a Gaussian shape for the wake deficit profile, the influence of an upwind turbine should be extended theoretically to all the downwind turbines regardless of their distance from the centre of the wake. However, if a downwind turbine is sufficiently far from the centre of the wake the velocity deficit due to the upwind turbine is negligible. Thus, it would be possible to avoid computing the streamwise distance $s_{i, j}$, the radial distance $r_{i, j}$ and the velocity deficit $\Delta U_{i, j} / U_{\infty}^{l o c}$, caused by the upwind turbine $j$ on the downwind turbine $i$, provided that turbine $i$ is located sufficiently far from the wake centerline of turbine $j$. All of these operations require significant computational time if they are repeated for a large number of iterations. Therefore, a considerable amount of time can be saved.

To enable this time save, an exclusion criterion that is simple, reliable and fast to implement is proposed. The criterion is simply to exclude areas with a 
velocity deficit less than 1\%. From Eq. (3) we get,

$$
\frac{\Delta U}{U_{\infty}^{l o c}}=C\left(s, U_{\infty}^{l o c}\right) \cdot \exp \left(-\frac{(r / D)^{2}}{2\left(k^{*} s / D+\epsilon\right)^{2}}\right)=0.01
$$

where

$$
C\left(s, U_{\infty}^{l o c}\right)=\left(1-\sqrt{1-\frac{C_{T}\left(U_{\infty}^{l o c}\right)}{8\left(k^{*} s / D+\epsilon\right)^{2}}}\right)
$$

Thus, the exclusion boundary $R_{\text {exc }}$ can be derived as a function of $s$ and $U_{\infty}^{l o c}$ :

$$
R_{e x c}\left(s, U_{\infty}^{\text {loc }}\right)=D \cdot \sqrt{2\left(k^{*} s / D+\epsilon\right)^{2} \cdot \ln \frac{C\left(s, U_{\infty}^{\text {loc }}\right)}{0.01}}
$$

The radius defined by this condition varies along the trajectory of the wake, as it can be seen in Fig. 11. Nevertheless, since the distances $s_{i, j}$ (denoting streamwise distance between the $i$ th and the $j$ th turbine) are not known in advance, it is assumed that the radius of the area of interest $R_{b}$ is constant and equal to the maximum $R_{\text {exc }}$ for the downwind distance ranging from $2 D$ to $40 D$. Thus, $R_{b}$ is defined as:

$$
R_{b}\left(U_{\infty}^{l o c}\right)=\max _{2 D \leq s \leq 40 D} R_{e x c}\left(s, U_{\infty}^{l o c}\right)
$$

Variation of $R_{e x c}$ and $R_{b}$ along the streamwise distance in the case of flat terrain is shown in Fig. 11.

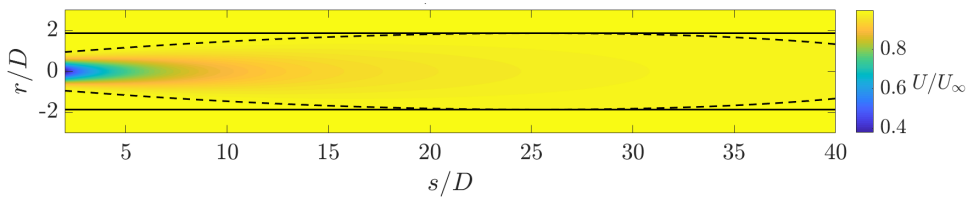

Figure 11: Variation of the boundary of the exclusion criterion $R_{\text {exc }} / D$ defined by the condition $\Delta U / U_{\infty}^{l o c}=0.01$ (dotted line), and the selected exclusion boundary $R_{b} / D$ obtained by setting a constant radius equal to the maximum one (solid line). $U_{\infty}^{l o c}=U_{\infty}=7 \mathrm{~m} / \mathrm{s}, C_{t}=0.747$.

By excluding wind turbines outside the area of interest defined by $R_{b}$, only the turbines with a velocity deficit of at least $1 \%$ caused by the upwind turbine under examination are taken into account. Some turbines, if any, with a slightly lower velocity deficit, that would be excluded by using a non-constant exclusive radius $R_{e x c}$ are selected as well, but the number of such turbines is usually very small, if not zero, and therefore the exclusion criterion remains very effective in excluding downwind turbines that are far from the centre of the wake.

An example demonstrating how the exclusion criterion works is shown in Fig. 12 , 


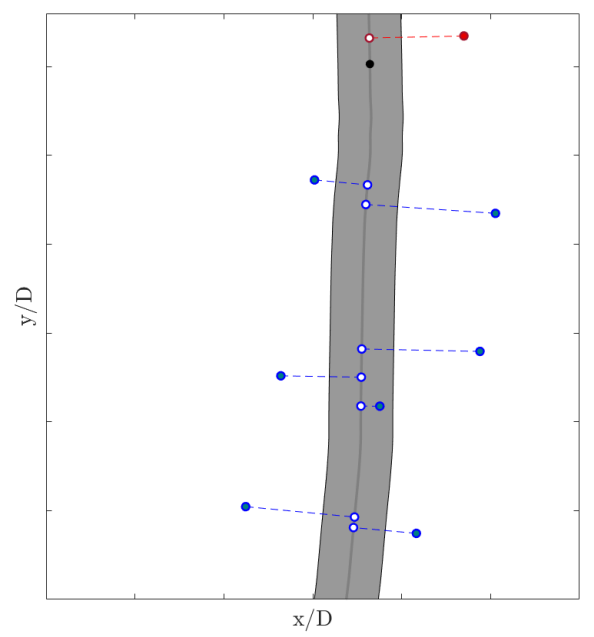

Figure 12: Exclusion criterion: only the turbines that are sufficiently close to the streamline that describe the wake centre trajectory are selected. At this stage no distinction is made between upwind (in red) and downwind turbines (in blue) with respect to the turbine whose wake is being analyzed (in black).

Following the above strategy, for any pair of turbines (the $i$ th and the $j$ th), it is possible to determine whether the $i$ th turbine is sufficiently influenced by the $j$ th turbine's wake, according to their relative position under a given inflow wind direction and the exclusive criterion defined by Eq. (9). Thus, the velocity deficit at the $i$ th turbine due to the $j$ th turbine's wake $\Delta U_{i, j}$ can be treated as 0 if there is no sufficient influence or computed with Eqs. (3) and (5) otherwise.

For a given reference inflow wind speed $U_{r e f}$ and a wind direction $\theta_{r e f}$, which are usually defined at the ideal far-field hub height level but can also be defined at a specific location, the local inflow freestream wind speed $U_{\infty}^{\text {loc }}$ will be different for different turbine locations in complex terrain. Denoting the local inflow wind speed $U_{\infty}^{\text {loc }}$ in the background flow field at the $i$ th turbine position as $U_{i}$, we can define the speed-up factor at the $i$ th turbine position with respect to the reference wind speed as $S_{i}=U_{i} / U_{\text {ref }}$. Then, following the balance of energy assumption proposed by Katic et al. [23] to account for the influence of multiple wakes, it is possible compute the effective wind speed at the $i$ th turbine in a wind farm with $N_{t}$ turbines as:

$$
\bar{U}_{i}=S_{i} U_{r e f}-\sqrt{\sum_{j=1}^{N_{t}}\left(\Delta U_{i, j}\right)^{2}}
$$

After the effective wind speeds at each wind turbine's rotor are calculated, the power outputs for each turbine and the whole wind farm can be easily computed from the turbine power curve. 


\subsection{Validation results}

The proposed wake model is based on several assumptions and its reliability in making accurate predictions has to be validated with other approaches. Two comparisons are performed: one with field measurement data from a SCADA system and the other with numerical data of high-fidelity CFD simulations. For the present case, the field measurement data is from a one-year field measurement campaign carried out in the studied wind farm, described and discussed in [46. The numerical results are from Reynolds Averaged Navier-Stokes (RANS) simulations by Sessarego et al. [40, where wind turbine wakes have been modelled by using the Actuator Disk (AD) concept.

As far as the power and thrust curves are concerned, in order to perform an unbiased comparison, the same curves used in the RANS/AD simulation have been adopted. The curves are displayed in Fig. 1 and differ slightly from the data provided by the manufacturer.

The model has been tested under different wind conditions: in particular two wind speeds $(7 \mathrm{~m} / \mathrm{s}$ and $10 \mathrm{~m} / \mathrm{s})$ and three directions $\left(180^{\circ}, 300^{\circ}\right.$ and $\left.330^{\circ}\right)$ have been studied for a total of six different combinations. A comparison has been made between the power measured by the SCADA system, the power predicted by the RANS/AD simulation, and the power predicted by the model proposed.

The different reference wind speeds and directions for the six scenarios are summarized in Table 2. The reference wind speed $\left(U_{r e f}\right)$ refers to the wind speed measured at hub height at turbine 11 or 14 depending on the cases, while the reference wind direction $\left(\theta_{\text {ref }}\right)$ is measured at met mast M1 or M3 at $70 \mathrm{~m}$ above the ground in the different inflow conditions. For details about the field measurements and the selection of reference wind directions, one is referred to [46].

Table 2: Inflow conditions for the six flow cases used in the validation of the proposed new wake model

. Reference wind speed $U_{\text {ref }}$ and reference wind direction $\theta_{\text {ref }}$ are measured at $67 \mathrm{~m}$ and $70 \mathrm{~m}$ height respectively. $\mathrm{WT}=$ wind turbine, $\mathrm{M}=$ met mast.

\begin{tabular}{ccccc}
\hline Flow Case & $\begin{array}{c}U_{\text {ref }} \\
{\left[{ }^{\circ}\right]}\end{array}$ & $\begin{array}{c}\theta_{\text {ref }} \\
{[\mathrm{m} / \mathrm{s}]}\end{array}$ & Speed reference & Direction reference \\
\hline 1 & 180 & 7 & WT14 & M3 \\
2 & 300 & 7 & WT11 & M1 \\
3 & 330 & 7 & WT11 & M1 \\
4 & 180 & 10 & WT14 & M3 \\
5 & 300 & 10 & WT11 & M1 \\
6 & 330 & 10 & WT11 & M1 \\
\hline
\end{tabular}

For each flow case, the wind speeds at the 25 turbines predicted by the proposed wake model are different from the SCADA measurement and the RANS/AD simulation. The means and the standard deviations of the relative differences for these 25 turbines are reported in Table 3. 
Table 3: Average relative difference and standard deviation of wind speeds at the 25 turbines of the studied wind farm under the six inflow conditions that are predicted by the proposed new wake model compared with those given by RANS/AD and SCADA data.

\begin{tabular}{|c|c|c|c|c|c|c|}
\hline \multirow{2}{*}{ Flow Case } & \multirow{2}{*}{$\begin{array}{c}\text { Wind Speed } \\
{[\mathrm{m} / \mathrm{s}]}\end{array}$} & \multirow{2}{*}{$\begin{array}{c}\text { Wind Direction } \\
{\left[^{\circ}\right]}\end{array}$} & \multicolumn{2}{|c|}{ RANS/AD } & \multicolumn{2}{|c|}{ SCADA } \\
\hline & & & $\begin{array}{c}\text { Mean } \\
{[\%]}\end{array}$ & $\begin{array}{c}\text { STD } \\
{[\%]}\end{array}$ & $\begin{array}{c}\text { Mean } \\
{[\%]}\end{array}$ & $\begin{array}{c}\text { STD } \\
{[\%]}\end{array}$ \\
\hline 1 & 7 & 180 & -2.43 & 7.32 & -12.73 & 24.98 \\
\hline 2 & 7 & 300 & -2.06 & 5.64 & -9.7 & 12.18 \\
\hline 3 & 7 & 330 & -3.25 & 3.08 & -4.31 & 24.7 \\
\hline 4 & 10 & 180 & -0.01 & 6.18 & -9.39 & 23.64 \\
\hline 5 & 10 & 300 & -0.07 & 4.23 & 6.26 & 13.52 \\
\hline 6 & 10 & 330 & -1.37 & 2.11 & 7.98 & 17.01 \\
\hline
\end{tabular}

On average the results appear to have a better agreement at angles of $300^{\circ}$ and $330^{\circ}$ and between the proposed model with RANS/AD. As suggested by Sessarego et al. 40, a possible explanation could be the non-uniform distribution for the incoming wind when coming from South $\left(180^{\circ}\right)$. The data from experimental measurements and the ones obtained through simulations can differ greatly. This large difference can be attributed to the uncertainty and error in field measurements [46], or the accuracy of the RANS/AD simulation [40], or more likely both.

Focusing on the results from the proposed model and the RANS/AD simulation, though, a modest difference can be noticed. This confirms the accuracy of the proposed model for modelling wind turbine wakes in complex terrain with respect to a high fidelity simulation. On the other hand, it puts in evidence the strong dependence the model has on CFD data to model the background flow field, which might cause significant differences with respect to the real flow.

\subsection{Summary}

As one of the main contributions in this study, the wake model described above is briefly summarized here with steps of using it. To model the effective wind speed all turbines experienced in the wind farm, the following procedure is taken:

- Step 1: Simulate the background flow field in the domain of interest for a number of wind direction sectors using CFD or any wind resource assessment tool.

- Step 2: Extract velocity components for all sectors at the iso-surface at hub height level above the ground and store the information on a grid covering the domain of interest.

- Step 3: Pre-compute a group of streamlines starting from the upwind boundary with uniform spacing for each sector, and store the information. 
- Step 4: For a given wind farm with specific layout and all wind direction sectors, determine the local inflow freestream wind speeds at each wind turbine based on the extracted background flow field information.

- Step 5: Calculate the wake deficits any turbine might cause on the other turbines using the superposition strategy described before and obtain the effective wind speed at each wind turbine for all sectors.

Although the validation results confirmed that the proposed new wake model can obtain results with a similar accuracy as high fidelity simulations such as RANS/AD, there are also certain limitations. The proposed model is based on the three assumptions. While these assumptions are made to approximate the reality reasonably well and also largely reduce the computational burden, they also bring some limitations to the model, which are listed as follows:

- The presence of turbine will alter the streamline from the rotor center, thus not directly following the background streamline.

- The pseudo 3D streamlines calculated following Assumption 2 is an approximation of the real 3D streamlines. The strategy to use a streamline parallel to the closest streamline among the set of pre-computed streamlines is an approximation and results in a minor error. However, this problem can be alleviated by pre-computing more streamlines.

- The Gaussian shape is a good approximation of reality, but it is not perfect, in particular it extends indefinitely, moreover it cannot be applied in the near wake region.

Nevertheless, comparing with the existing engineering wake models, the proposed new model is still the wake model most suitable for applications in complex terrain.

\section{Layout optimization}

\subsection{Problem formulation}

Assuming a wind farm composed of $N_{t}$ wind turbines of the same type, the layout is defined with the $x$ and $y$ coordinates of the positions of the turbines, i.e., $\mathbf{X}=\left[x_{1}, x_{2}, \ldots, x_{N_{t}}\right], \mathbf{Y}=\left[y_{1}, y_{2}, \ldots, y_{N_{t}}\right]$. Then the layout optimization problem is formulated as maximizing or minimizing an objective function $f(\mathbf{X}, \mathbf{Y})$, while subject to some requirements and/or constraints on the design variables, $\mathbf{X}$ and $\mathbf{Y}$. The most widely used objective functions include: power output, annual energy production (AEP), levelized cost of energy (LCOE), net present value or other financial metrics of the wind farm project. Commonly included constraints are boundaries, exclusive zones, and minimal distance requirements.

Many algorithms have been proposed to solve this problem, while systematic comparisons among different algorithms have rarely been done. A direct 
comparison between different studies also suffers from the challenge brought by differences in problem formulation, details in wake modelling and the studied cases.

In this study, the main purpose is to systematically compare the performance of several algorithms for a wind farm layout optimization problem in complex terrain, based on the proposed wake model. Considering this purpose, we formulate the layout optimization problem with a simplified objective function, i.e., the sum of normalized effective wind speeds at all wind turbines, averaged over all wind direction sectors. Hence, the objective function can be written as:

$$
\max \sum_{w=1}^{N_{d}} \sum_{i=1}^{N_{t}} p_{i, w} \frac{\bar{U}_{i, w}}{U_{r e f}}
$$

where $N_{d}$ represents the number of wind direction sectors considered, $p_{i, w}$ denotes the probability of wind sector $w$ at the location of the $i$ th turbine and $\bar{U}_{i, w}$ is the effective wind speed at turbine $i$ under inflow wind sector $w$. Note that $\bar{U}_{i, w}$ can be calculated using Eq. 10 based on the proposed wake model.

Constraints on the minimal distance between any two turbines and boundary set by the rectangular domain shown in Fig. $2 \mathrm{~b}$ are considered, which are governed by:

$$
\begin{gathered}
\sqrt{\left(x_{i}-x_{j}\right)^{2}+\left(y_{i}-y_{j}\right)^{2}} \geq 4 D, \forall i, j \in\left\{1,2, \ldots, N_{t}\right\} \text { and } i \neq j \\
0 \leq x_{i} \leq 6000 m, 0 \leq y_{i} \leq 4000 m, \forall i \in\left\{1,2, \ldots, N_{t}\right\}
\end{gathered}
$$

\subsection{Optimization algorithms}

Optimization is a broad field that covers a large spectrum of optimization algorithms, each has its advantages and shortages and suits some specific kinds of optimization problems [47. In the field of wind farm layout optimization, various types of optimization algorithms have been applied [4, including generalpurpose algorithms (such as genetic algorithm [48] and particle swarm optimization 49) and specifically developed algorithms (such as random search 19]).

According to whether the gradient information is used, optimization algorithms can be broadly divided into two types: gradient-based and gradient-free algorithms [47. While gradient-based algorithms can be very effective in finding the local minimum, they usually require certain mathematical properties of the problem, such as continuous and differentiable objective functions. They also encounter problems in finding the global minimum when the design variable space is high dimensional and highly nonlinear, as is the case for wind farm layout optimization. Besides, for cases without gradients in analytical form, the required gradient information will have to be computed by numerical approximation, which also increases the computational cost at each step. For example, Guirguis et al. 50] tried to optimize the wind farm layout using a classical nonlinear mathematical programming method by simplifying the ob-

jective function and utilizing the gradient. On the other hand, gradient-free 
algorithms are more robust and easier to implement for solving complicated optimization problems, as they only require calculating the objective and constraint functions. Although these algorithms tend to converge slower than their gradient-based counterparts, they usually have a higher probability to find the global optimum. Thus, most of the studies on wind farm layout optimization employed this type of algorithms.

Based on the number of solutions kept during the optimization process, an optimization algorithm can be either single solution based or population based. For single solution based algorithms, only one solution is kept and iteratively improved during the optimization process, while the population based algorithms maintain a group of solutions at each step. Genetic algorithm is one famous example of population based algorithms 48.

In this study, eight optimization algorithms are compared. Their characteristics are summarized in Table 4

Table 4: Characteristics of the eight optimization algorithms considered in the comparative study.

\begin{tabular}{lcccc}
\hline Name & Gradient & Solution size & Implementation & Ref. \\
\hline Global search (GS) & Yes & Single & Matlab toolbox & {$[51]$} \\
MultiStart (MS) & Yes & Single & Matlab toolbox & {$[52]$} \\
Pattern search (PS) & No & Single & Matlab toolbox & {$[53]$} \\
Genetic algorithm (GA) & No & Population & Matlab toolbox & {$[48$} \\
Particle swarm optimization (PSO) & No & Population & Matlab toolbox & {$[49]$} \\
Simulated annealing (SA) & No & Single & Matlab toolbox & {$[54]$} \\
Local search (LS) & No & Single & Self-made & {$[18$} \\
Random search (RS) & No & Single & Self-made & {$[19]$} \\
\hline
\end{tabular}

Except for LS and RS, all the algorithms are general-purpose optimization methods. The first six algorithms applied here are all provided by Matlab in its Global Optimization toolbox are applied here. As a widely used commercial software, the Global Optimization toolbox is a good choice for comparing different algorithms, as it provides mature and professional implementations of the optimization algorithms. Furthermore, the algorithms implemented in the Matlab toolbox, with the exception of PSO and SA, can accept user defined nonlinear constraints and bounds on design variables. Thus, they can naturally handle the constraints considered in this study. For PSO and SA a penalty function was added to the objective function to take into account violations of the non-linear constraints. Details of the algorithms and their implementations can be found in the references listed in Table 4 and in the manual of the Global Optimization toolbox [52.

The LS algorithm is an optimization algorithm proposed specifically for wind farm layout optimization. It was introduced by Wagner et al. in 2013 [18] and is fast, effective and easy to implement. The algorithm makes large use of randomness to search for the global minimum. The basic idea is to move a single turbine at a time: if the move improves the objective function the new 
location is accepted, otherwise it is rejected. The displacement of the turbine is based on randomness, but the information regarding the position of the closest turbines and past moves is used as well. Randomness allows exploring the search space by escaping local minima, while the information regarding closest turbines and previous good moves allow refining the search close to a minimum.

The RS algorithm was proposed by Feng and Shen [19]. It improves the wind farm layout iteratively in a similar manner as the LS algorithm, i.e., by moving a randomly chosen turbine from its position to a new position at each step. The difference between RS and LS lies in their way of determining the new position. While LS uses information regarding the positions of neighbouring turbines and past moves, RS chooses a purely randomly determined position. Details of these two algorithms are referred to the references [19, 18.

In this study, we implement the LS and RS algorithm in Matlab, following the procedure described in the original studies [19, 18], together with the parameters recommended there.

\subsection{Double-stage approach}

For high dimensional and complex optimization problems, choosing a good initial solution is usually an important task that will influence the quality of the final results. For example, gradient-based algorithms tend to converge to different local minimums when starting from different initial solutions [47.

Starting from diverse initial solutions is one way to tackle this problem, as adopted by the MS algorithm. Combining two algorithms and seeding results from one algorithm to the other as the initial solution is another way, as have been tried by Saavedra-Moreno et al. [55.

To utilize the non-uniform nature of complex terrain sites, a simple doublestage approach for optimizing wind farm layout is proposed here. This approach can be applied for wind farm design in complex terrain with any objective function that involves wake modelling, such as wind speed (as in our case), power or AEP. The following two stages of optimization are carried out in order for all of the optimization algorithms:

- Stage 1: Optimize the objective function without considering wake effects.

- Stage 2: Starting from the optimized solution(s) of the first stage, optimize the objective function considering wake effects.

Following the problem formulation adopted in this study, the objective function taken in Stage $\mathbf{1}$ is thus governed by:

$$
\max \sum_{w=1}^{N_{d}} \sum_{i=1}^{N_{t}} p_{i, w} \frac{U_{i, w}^{\text {nowake }}}{U_{\text {ref }}} \Rightarrow \max \sum_{w=1}^{N_{d}} \sum_{i=1}^{N_{t}} p_{i, w} S_{i, w}
$$

In this double-stage approach, the first stage optimization can be done very quickly as it avoids the computationally heavy wake modelling. Nevertheless, it can still generate quite good starting layouts for the second stage, as it tries 
to place turbines in sites with good wind resources. The second stage can then build on the starting layouts to yield better final results than a conventional single-stage approach, under the same computational time limit. Note that this approach only works for complex terrain sites since wind farms on flat terrain or at offshore sites experience more or less uniform wind resource, which makes the first stage optimization meaningless.

\section{Results}

The optimization algorithms listed in the previous section are tested for the layout optimization problem of the studied wind farm as described in Section 2. The objective function and constraints have been introduced in Section 4 .

For the test case used here, the reference wind speed is set as $U_{\text {ref }}=7$ $\mathrm{m} / \mathrm{s}$, representing the far-field ideal inflow. Twelve wind direction sectors are considered. The centres of these sectors correspond to the wind directions of $\left[0^{\circ}, 30^{\circ}, \ldots, 330^{\circ}\right]$.

To compare the performance of different algorithms, a fixed time limit is set for each optimization run, although the actual execution time can be longer or shorter, due to the computation of the last step or generation, or the premature convergence before the time limit. This time limit is set as 20000 seconds for runs with the single-stage approach, and 25000 seconds for runs with the doublestage approach. Note that the time considered here is the consumed CPU time of running the algorithm on a computer with a $3.2 \mathrm{GHz} \mathrm{CPU}$ and $16 \mathrm{~GB}$ of RAM.

In the current study, the objective function is only continuous if the exclusion criterion presented in sub-section 3.3 is not applied. The exclusion criterion is certainly helpful in reducing the computation time by decreasing the number for which certain computations must be performed. However, it introduces discontinuities by setting the velocity deficit equal to zero when a turbine is moved outside of the exclusion boundary of the wake. Although the discontinuities are small, they pose serious problems to algorithms that are gradient-based and therefore require a continuous and differentiable objective function. As a consequence, for the two tested gradient-based algorithms, i.e., MS and GS, the exclusion criterion is not used.

First, the algorithms are tested using the conventional one-stage approach, where the original layout is used as the initial solution and the time limit for each run is 20000 seconds. The results are summarized in Table 5 .

Table 5: Comparison of the eight algorithms' performance starting from the original layout.

\begin{tabular}{|c|c|c|c|c|c|c|c|c|}
\hline \multirow{2}{*}{ Results } & \multicolumn{2}{|c|}{ Gradient-based } & \multicolumn{6}{|c|}{ Gradient-free } \\
\hline & MS & GS & PS & GA & PSO & $\mathrm{SA}$ & LS & $\mathrm{RS}$ \\
\hline Objective function [-] & 27.38 & 27.40 & 27.75 & 27.14 & 27.27 & 27.26 & 28.02 & 27.95 \\
\hline Relative change $[\%]^{*}$ & 1.86 & 1.93 & 3.24 & 0.97 & 1.45 & 1.41 & 4.24 & 3.98 \\
\hline
\end{tabular}

${ }^{*}$ Computed with respect to the original layout with an objective function of 26.88 . 
As shown by Table 5, the gradient-based algorithms, MS and GS, show a better performance than three of the gradient-free algorithms (GA, PSO and GA), but are outperformed by PS, LS and RS. Among the eight algorithms, LS and RS are the top two in terms of improvement of the objective function. It is also worthy to note that the initial solution used here, i.e., the original layout of the studied wind farm, is already a quite good one, as it was chosen by the developer after some optimization studies.

After examining the ability of the algorithms to improve the current wind farm layout, they are tested on random layouts to understand if these algorithms can find an optimal solution by starting from a random layout, rather than from an already known good starting solution. Ten different layouts that satisfy the constraints are randomly generated and the algorithms are applied starting from these layouts.

Both the single-stage approach and the double-stage approach are taken. For the single-stage approach, the time limit for each optimization run is also set as 20000 seconds. For the double-stage approach, however, the time limit is set as 25000 seconds, which uses 5000 seconds for stage 1 and 20000 seconds for stage 2 .

Results using the single-stage approach are reported in Table 6, which includes the statistics of ten runs of the algorithms from the ten different random initial solutions.

Table 6: single-stage approach: comparison of the eight algorithms' performance starting from ten random layouts (average objective function value of these ten layouts is 24.90).

\begin{tabular}{|c|c|c|c|c|c|c|c|c|}
\hline \multirow{2}{*}{ Statistics } & \multicolumn{2}{|c|}{ Gradient-based } & \multicolumn{6}{|c|}{ Gradient-free } \\
\hline & MS & GS & PS & GA & PSO & $\mathrm{SA}$ & LS & $\mathrm{RS}$ \\
\hline \multicolumn{9}{|l|}{ Mean } \\
\hline Objective function [-] & 26.85 & 26.78 & 27.72 & 26.52 & 27.22 & 26.85 & 27.99 & 28.01 \\
\hline Relative change $[\%]^{*}$ & -0.11 & -0.37 & 3.13 & -1.34 & 1.26 & -0.11 & 4.13 & 4.20 \\
\hline \multicolumn{9}{|l|}{ Min } \\
\hline Objective function [-] & 26.56 & 26.33 & 27.64 & 26.26 & 26.77 & 26.44 & 27.94 & 27.93 \\
\hline Relative change $[\%]^{*}$ & -1.19 & -2.05 & 2.83 & -2.31 & -0.41 & -1.64 & 3.94 & 3.91 \\
\hline \multicolumn{9}{|l|}{ Max } \\
\hline Objective function [-] & 27.07 & 27.16 & 27.83 & 26.77 & 27.55 & 27.10 & 28.07 & 28.10 \\
\hline Relative change $[\%]^{*}$ & 0.71 & 1.04 & 3.53 & -0.41 & 2.49 & 0.82 & 4.43 & 4.54 \\
\hline Standard Deviation & & & & & & & & \\
\hline Objective function [-] & 0.16 & 0.30 & 0.06 & 0.15 & 0.21 & 0.19 & 0.04 & 0.05 \\
\hline
\end{tabular}

On average, the best results are also obtained by the RS and LS algorithms in this case. RS and LS are the only two algorithms to exceed the value of 28 for the objective function and to have good layouts systematically. Even 
in the worst case, the objective function value was 27.93 for RS and 27.94 for LS. Comparing LS with RS, we can notice that their performance are quite similar in general, while RS achieves slightly better results for the mean and maximum objective function. PS obtained the third highest results and it did so with slight variations, as it can be observed by the low standard deviation, comparable to the ones of RS and LS. PS is the only algorithm, along with RS, LS and PSO, that obtained objective function values over 27 on average. All the other algorithms obtained results in line with the objective function of the original layout, with the exception of GA, which obtained the worst results in terms of objective function value.

Two gradient-based algorithms, MS and GS, did not obtain good layouts because they rely on the quality and number of the starting solutions. Being gradient-based solvers, their performance in terms of time of execution is worse due to the additional time required to compute gradients. Moreover, by not making use of the exclusion criterion to avoid discontinuities, the computation time for a single iteration is also higher since more computations are required. Thus, they cannot run as many iterations as the other gradient-free algorithms under the same total time limit, which limits their performance in terms of the optimized objective function.

Similarly, Table 7 shows the results using the double-stage approach.

Table 7: double-stage approach: comparison of the eight algorithms' performance starting from ten random layouts (average objective function value of these ten layouts is 24.90).

\begin{tabular}{|c|c|c|c|c|c|c|c|c|}
\hline \multirow{2}{*}{ Statistics } & \multicolumn{2}{|c|}{ Gradient-based } & \multicolumn{6}{|c|}{ Gradient-free } \\
\hline & MS & GS & PS & GA & PSO & $\mathrm{SA}$ & LS & $\mathrm{RS}$ \\
\hline \multicolumn{9}{|l|}{ Mean } \\
\hline Objective function [-] & 26.97 & 27.03 & 27.80 & 26.93 & 27.66 & 27.05 & 28.03 & 28.04 \\
\hline Relative change $[\%]^{*}$ & 0.33 & 0.56 & 3.42 & 0.19 & 2.90 & 0.63 & 4.28 & 4.32 \\
\hline \multicolumn{9}{|l|}{ Min } \\
\hline Objective function [-] & 26.89 & 26.93 & 27.67 & 26.69 & 27.50 & 26.74 & 27.97 & 28.00 \\
\hline Relative change $[\%]^{*}$ & 0.04 & 0.19 & 2.94 & -0.71 & 2.31 & -0.52 & 4.06 & 4.17 \\
\hline \multicolumn{9}{|l|}{ Max } \\
\hline Objective function [-] & 27.11 & 27.22 & 27.92 & 27.65 & 27.76 & 27.74 & 28.09 & 28.07 \\
\hline Relative change $[\%]^{*}$ & 0.86 & 1.26 & 3.87 & 2.86 & 3.27 & 3.20 & 4.50 & 4.43 \\
\hline Standard Deviation & & & & & & & & \\
\hline Objective function [-] & 0.08 & 0.11 & 0.09 & 0.34 & 0.09 & 0.35 & 0.04 & 0.02 \\
\hline
\end{tabular}

From a brief comparison with the single-stage approach results, it can be noticed that all the algorithms show an improvement in their performance in general. This can be partially explained by the longer time available, but also by the better initial layout provided by the first stage. The only exception is for 
RS, which obtains a lightly better maximum objective function using the singlestage approach than using the double-stage approach (28.10 versus 28.07). With the double-stage approach all the algorithms, except for MS and GA, have an average objective function higher than 27. RS and LS obtain objective function values on average over 28, which are quite remarkable results. In this case, though, the longer time available might be the main reason to explain the better result.

The algorithms that showed most improvements are those that showed the worse results, in particular GA and SA, but also PSO improved its final results significantly and reduced the standard deviation. SA achieved a high maximum value, but the large standard deviation shows how this is not a consistent result, the minimum value is rather low indeed. PS, having already extremely satisfying results, showed a limited improvement in its final objective function value. MS and GS have only slightly better final layouts, instead.

To better visualize the effectiveness of the double-stage approach, the comparison of results of single and double-stage approaches is shown in Fig. 13.

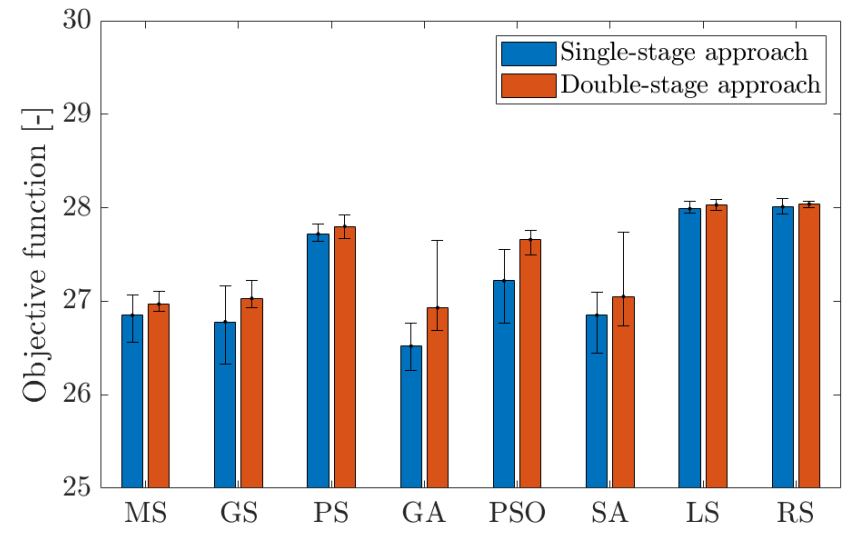

Figure 13: Comparison of results using single and double-stage approaches. The mean value along with the minimum and maximum are displayed for each algorithm.

The best layouts found by different algorithms in this study are reported in Table 8 .

Table 8: Comparison of best layouts found by the eight algorithms.

\begin{tabular}{|c|c|c|c|c|c|c|c|c|}
\hline \multirow{2}{*}{ Results } & \multicolumn{2}{|c|}{ Gradient-based } & \multicolumn{6}{|c|}{ Gradient-free } \\
\hline & MS & GS & PS & GA & PSO & SA & $\mathrm{LS}$ & $\mathrm{RS}$ \\
\hline Objective function [-] & 27.38 & 27.40 & 27.92 & 27.65 & 27.76 & 27.74 & 28.09 & 28.10 \\
\hline Relative change [\%] & 1.86 & 1.93 & 3.87 & 2.86 & 3.27 & 3.20 & 4.50 & 4.54 \\
\hline
\end{tabular}

${ }^{*}$ Computed with respect to the original layout with an objective function of 26.88 . 
In general, RS and LS are the best performing algorithms, which achieve the best results while show least variations in multiple runs (as suggested by the low standard deviation values). The best layouts found by the gradientfree algorithms are usually better than the those determined by gradient-based algorithms, among which the best layout found by RS achieves the highest objective function. This layout found by RS is shown in Fig. 14.

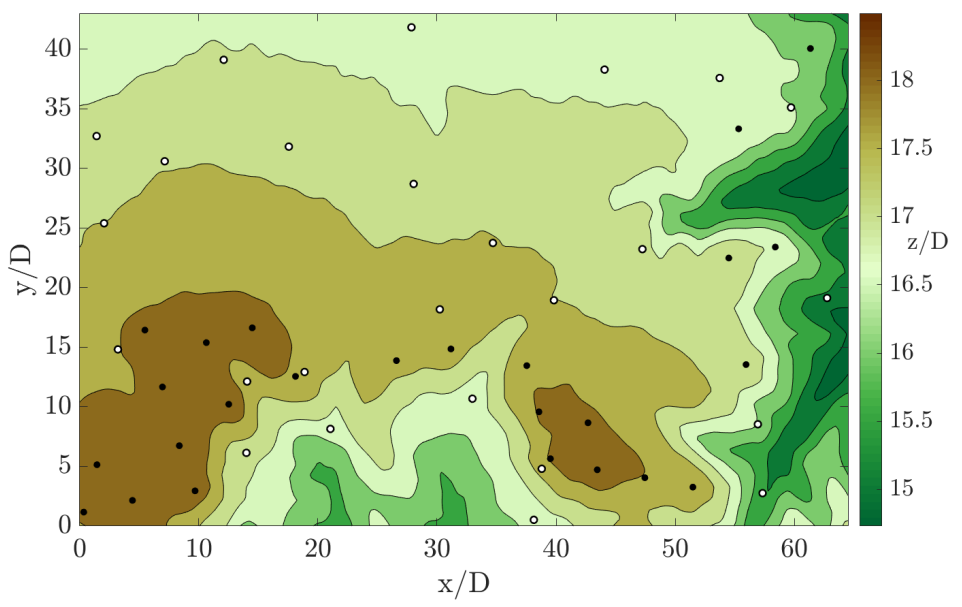

Figure 14: Best performing layout found by RS using the single-stage approach. The initial random layout (white circles) is compared with the final layout (black circles).

\section{Conclusions}

Determining a good layout of the initial position of wind turbines is of central importance for the success of a wind farm project. This task is extremely challenging for wind farms in complex terrain, largely due to the difficulties in handling wake modelling. While high fidelity numerical methods such as CFD are capable of simulating such flows reasonably well, the high computational costs make them unsuitable for direct applications in layout optimization.

One of the main contributions of this study is the development of an new engineering wake model that can model the wake flow in real complex terrain sites with reasonable accuracy in a short time. This model is built on superposing a state-of-the-art Gaussian shape engineering wake model for wind turbines on flat terrain on top of the background flow field in complex terrain, i.e., flow fields without wind turbines, obtained by CFD simulations. The centerlines of wakes of the wind turbine are assumed to follow the streamlines in the background flow field.

A fast approach to calculate the streamlines in the background flow field from any turbine rotor centre position is also proposed. To use this approach, only one layer of velocity information on the iso-surface at hub height level above the ground is needed. Based on the velocity information, a group of streamlines 
in the background flow field can be pre-computed and stored in advance for each wind direction sector, which enables fast calculations of wake effects between any turbines on the fly during the optimization. In order to further reduce the computation time, an exclusion criterion is proposed to exclude computations of negligible wake effects between turbines. The effectiveness of the proposed wake model has been validated against CFD simulation (RANS/AD) and SCADA data for a real wind farm in complex terrain.

To demonstrate the usage of the proposed wake model, we also studied the layout optimization problem for a real wind farm with 25 turbines located at a complex terrain site in Northwest China.

Although there have been a large number of algorithms, both general-purpose ones or specifically developed ones, applied to wind farm layout optimization, systematically comparative studies on optimization algorithms are largely missing. Considering this fact, in this study we choose to focus on the systematic comparison of different algorithms in the context of layout optimization for wind farms in complex terrain, with the objective simplified as maximizing the sum of the normalized wind speeds.

Eight algorithms are applied and compared in this study, including MultiStart, GlobalSearch, Pattern Search, Genetic Algorithm, Particle Swarm Optimization, Simulated Annealing, Local Search and Random Search. A simple double-stage approach was also proposed to obtain better results when optimizing wind farm layout in complex terrain, which divides the optimization process into two stages: a stage in which the objective function is optimized without considering wake effects, followed by a stage which optimizes the normal objective function with wake effects.

All the tested algorithms improved the original layout. Despite this, the algorithms showed significant differences in terms of final results. The algorithms that provide the best performance are Random Search and Local Search, followed by Pattern Search and Particle Swarm Optimization, while Simulated Annealing, Genetic Algorithm, MultiStart and GlobalSearch experienced the worse results. The algorithms proved to be suitable for improving both the original wind farm layout and some randomly generated layouts. The doublestage approach also demonstrated its effectiveness in getting better results than the conventional single-stage approach.

In summary, the new wake model developed in this study showed promising results in the validation study, thus can be used in layout optimizations of real-life wind farms in complex terrain. The systematic comparative study of eight optimization algorithms also provided useful insights for choosing proper algorithms when optimizing wind farm layouts.

Future work will include the proposed wake model and double-stage approach in an overall wind farm design framework and apply it to optimize more realistic objective functions, such as AEP and LCOE, while considering more constraints that might come in a real-life wind farm project. 


\section{Acknowledgements}

This work was supported by the Sino-Danish cooperative project Wind farm layout optimization in complex terrain funded by the Danish Energy Agency (EUDP J.nr. 64013-0405) and the CCA project TopFarm funded by the Department of Wind Energy, Technical University of Denmark.

\section{References}

[1] GWEC, Global wind report 2018, https://gwec.net/wp-content/ uploads/2019/04/GWEC-Global-Wind-Report-2018.pdf (May 2019).

[2] M. Samorani, The wind farm layout optimization problem, in: Handbook of wind power systems, Springer, 2013, pp. 21-38. doi:10.1007/ 978-3-642-41080-2_2

[3] G. Mosetti, C. Poloni, B. Diviacco, Optimization of wind turbine positioning in large windfarms by means of a genetic algorithm, Journal of Wind Engineering and Industrial Aerodynamics 51 (1994) 105-116. doi:10.1016/0167-6105(94)90080-9.

[4] J. S. González, M. B. Payán, J. M. R. Santos, F. González-Longatt, A review and recent developments in the optimal wind-turbine micro-siting problem, Renewable and Sustainable Energy Reviews 30 (2014) 133-144. doi:10.1016/j.rser.2013.09.027

[5] Y. Chen, H. Li, K. Jin, Q. Song, Wind farm layout optimization using genetic algorithm with different hub height wind turbines, Energy Conversion and Management 70 (2013) 56-65. doi:10.1016/j.enconman.2013. 02.007

[6] A. Vasel-Be-Hagh, C. Archer, Wind farm hub height optimization, Applied Energy 195 (2017) 905-921. doi:10.1016/j.apenergy.2017.03.089.

[7] J. Feng, W. Z. Shen, Design optimization of offshore wind farms with multiple types of wind turbines, Applied Energy 205 (2017) 1283-1297. doi:10.1016/j.apenergy.2017.08.107.

[8] X. Gao, H. Yang, L. Lu, Study on offshore wind power potential and wind farm optimization in Hong Kong, Applied Energy 130 (2014) 519-531. doi : $10.1016 / j$.apenergy. 2014.02.070

[9] J. Park, K. Law, Layout optimization for maximizing wind farm power production using sequential convex programming, Applied Energy 151 (2015) 320-334. doi:10.1016/j.apenergy.2015.03.139.

[10] X. Gao, H. Yang, L. Lu, Optimization of wind turbine layout position in a wind farm using a newly-developed two-dimensional wake model, Applied Energy 174 (2016) 192-200. doi:10.1016/j.apenergy.2016.04.098. 
[11] J. S. González, M. B. Payán, J. M. Riquelme-Santos, Optimization of wind farm turbine layout including decision making under risk, IEEE Systems Journal 6 (2012) 94-102. doi:10.1109/JSYST.2011.2163007.

[12] M. Mayo, M. Daoud, Aesthetic local search of wind farm layouts, Information 8 (2017) 39. doi:10.3390/info8020039.

[13] J. Feng, W. Z. Shen, Wind farm power production in the changing wind: Robustness quantification and layout optimization, Energy Conversion and Management 148 (2017) 905-914. doi:10.1016/j.enconman.2017.06. 005 .

[14] X. Ju, F. Liu, Wind farm layout optimization using self-informed genetic algorithm with information guided exploitation, Applied Energy 248 (2019) 429-445. doi:10.1016/j.apenergy . 2019.04.084.

[15] B. L. Du Pont, J. Cagan, An extended pattern search approach to wind farm layout optimization, Journal of Mechanical Design 134 (2012) 081002. doi:10.1115/1.4006997.

[16] S. Chowdhury, J. Zhang, A. Messac, L. Castillo, Unrestricted wind farm layout optimization (UWFLO): Investigating key factors influencing the maximum power generation, Renewable Energy 38 (2012) 16-30. doi: 10.1016/j.renene.2011.06.033.

[17] U. A. Ozturk, B. A. Norman, Heuristic methods for wind energy conversion system positioning, Electric Power Systems Research 70 (2004) 179-185. doi:10.1016/j.epsr.2003.12.006

[18] M. Wagner, J. Day, F. Neumann, A fast and effective local search algorithm for optimizing the placement of wind turbines, Renewable Energy 51 (2013) 64-70. doi:10.1016/j.renene.2012.09.008.

[19] J. Feng, W. Z. Shen, Solving the wind farm layout optimization problem using random search algorithm, Renewable Energy 78 (2015) 182-192. doi: 10.1016/j.renene.2015.01.005.

[20] E. S. Politis, J. Prospathopoulos, D. Cabezon, K. S. Hansen, P. K. Chaviaropoulos, R. J. Barthelmie, Modeling wake effects in large wind farms in complex terrain: the problem, the methods and the issues, Wind Energy 15 (1) (2012) 161-182. doi:10.1002/we.481.

[21] F. Castellani, M. Astolfi, D.and Mana, E. Piccioni, M. Becchetti, L. Terzi, Investigation of terrain and wake effects on the performance of wind farms in complex terrain using numerical and experimental data, Wind Energy 20 (2017) 1277-1289. doi:10.1002/we.2094.

[22] X. Gao, T. Wang, B. Li, H. Sun, H. Yang, Z. Han, Y. Wang, F. Zhao, Investigation of wind turbine performance coupling wake and topography effects based on lidar measurements and scada data, Applied Energy 255 (2019) 113816. doi:10.1016//.apenergy.2019.114025 
[23] I. Katic, J. Højstrup, N. O. Jensen, A simple model for cluster efficiency, in: European wind energy association conference and exhibition, 1987, p. 4.

[24] R. Shakoor, M. Hassan, A. Raheem, Y.-K. Wu, Wake effect modeling: A review of wind farm layout optimization using jensen's model, Renewable and Sustainable Energy Reviews 58 (2016) 1048-1059. doi:10.1016/j. rser.2015.12.229.

[25] C. Archer, A. Vasel-Be-Hagh, C. Yan, S. Wu, Y. Pan, J. Brodie, A. Maguire, Review and evaluation of wake loss models for wind energy applications, Applied Energy 226 (2018) 1187-1207. doi:10.1016/j. apenergy.2018.05.085.

[26] M. Bastankhah, F. Porté-Agel, A new analytical model for wind-turbine wakes, Renewable Energy 70 (2014) 116-123. doi:10.1016/j.renene. 2014.01.002.

[27] N. Kirchner-Bossi, F. Porté-Agel, Realistic wind farm layout optimization through genetic algorithms using a gaussian wake model, Energies 11 (2018) 3268. doi:10.3390/en11123268.

[28] H. Sun, H. Yang, Study on an innovative three-dimensional wind turbine wake model, Applied Energy 226 (2018) 483-493. doi:10.1016/j. apenergy.2018.06.027.

[29] Y. Cheng, M. Zhang, Z. Zhang, J. Xu, A new analytical model for wind turbine wakes based on monin-obukhov similarity theory, Applied Energy (2019) 96-106doi:10.1016/j.apenergy .2019.01.225

[30] Z. Ti, X. W. Deng, H. Yang, Wake modeling of wind turbines using machine learning, Applied Energy 257 (2020) 114025. doi:10.1016//.apenergy. 2019.114025.

[31] M. Song, K. Chen, Z. He, X. Zhang, Bionic optimization for micro-siting of wind farm on complex terrain, Renewable Energy 50 (2013) 551-557. doi:10.1016/j.renene.2012.07.021.

[32] M. Song, K. Chen, Z. He, X. Zhang, Optimization of wind farm micrositing for complex terrain using greedy algorithm, Energy 67 (2014) 454459. doi:10.1016/j .energy . 2014.01.082.

[33] J. Feng, W. Shen, Wind farm layout optimization in complex terrain: A preliminary study on a gaussian hill, Journal of Physics: Conference Series 524 (1) (2014) 012146. doi:10.1088/1742-6596/524/1/012146.

[34] J. Feng, W. Z. Shen, Y. Li, An optimization framework for wind farm design in complex terrain, Applied Sciences 8 (2018) 2053. doi:10.3390/ app8112053. 
[35] J. Kuo, D. Romero, J. Beck, C. Amon, Wind farm layout optimization on complex terrains integrating a CFD wake model with mixedinteger programming, Applied Energy 178 (2016) 404-414. doi:10.1016/ j.apenergy.2016.06.085.

[36] S. Shamsoddin, F. Porté-Agel, Wind turbine wakes over hills, Journal of Fluid Mechanics 855 (2018) 671-702. doi:10.1017/jfm.2018.653.

[37] L. J. Vermeer, J. N. Sørensen, A. Crespo, Wind turbine wake aerodynamics, Progress in aerospace sciences 39 (6-7) (2003) 467-510. doi:10.1016/ S0376-0421(03) 00078-2.

[38] S. Frandsen, R. Barthelmie, S. Pryor, O. Rathmann, S. Larsen, J. Højstrup, M. Thøgersen, Analytical modelling of wind speed deficit in large offshore wind farms 9 (2006) 39-59. doi:10.1002/we.189.

[39] X. Yang, M. Pakula, F. Sotiropoulos, Large-eddy simulation of a utilityscale wind farm in complex terrain, Applied Energy 229 (2018) 767-777. doi:10.1016/j.apenergy.2018.08.049.

[40] M. Sessarego, W. Z. Shen, M. van der Laan, K. Hansen, W. J. Zhu, CFD simulations of flows in a wind farm in complex terrain and comparisons to measurements, Applied Sciences 8 (2018) 788. doi:10.3390/app8050788.

[41] H. Schlichting, Boundary Layer Theory, 7th Edition, McGraw-Hill, 1979.

[42] C. L.P., F. Porté-Agel, A wind-tunnel investigation of wind-turbine wakes: boundary-layer turbulence effects, Boundary-Layer Meteorology 132 (2009) 129-149. doi:10.1007/s10546-009-9380-8

[43] M. Gaumond, P. Réthoré, S. Ott, A. Pena, A. Bechmann, K. Hansen, Evaluation of the wind direction uncertainty and its impact on wake modeling at the horns rev offshore wind farm, Wind Energy 17 (2014) 11691178. doi:10.1002/we.1625,

[44] F. Carbajo Fuertes, C. Markfort, F. Porté-Agel, Wind turbine wake characterization with nacelle-mounted wind lidars for analytical wake model validation, Remote Sensing 10 (2018) 668. doi:10.3390/rs10050668.

[45] DTU Wind Energy, WAsP CFD, http://www.wasp.dk/waspcfd (May 2019).

[46] K. Hansen, G. Larsen, R. Menke, N. Vasiljevic, N. Angelou, J. Feng, W. Zhu, A. Vignaroli, W. Liu, C. Xu, W. Shen, Wind turbine wake measurement in complex terrain, Journal of Physics: Conference Series 753 (3) (2016) 032013. doi:10.1088/1742-6596/753/3/032013.

[47] M. French, Fundamentals of Optimization: Methods, Minimum Principles, and Applications for Making Things Better, Springer, 2018. 
[48] J. H. Holland, Adaptation in Natural and Artificial Systems: An Introductory Analysis with Applications to Biology, Control, and Artificial Intelligence, MIT Press, 1975.

[49] J. Kennedy, R. Eberhart, Particle swarm optimization, in: IEEE International Conference on Neural Networks, Vol. 4, 1995, pp. 1942-1948.

[50] D. Guirguis, D. Romero, C. Amon, Toward efficient optimization of wind farm layouts: Utilizing exact gradient information, Applied Energy 179 (2016) 110-123. doi:10.1016/j.apenergy.2016.06.101.

[51] Z. Ugray, L. Lasdon, J. Plummer, F. Glover, J. Kelly, R. Marti, Scatter search and local NLP solvers: A multistart framework for global optimization, INFORMS Journal on Computing 19 (3) (2007) 328-340. doi:10.1287/ijoc.1060.0175.

[52] The MathWorks Inc., Global Optimization Toolbox User's Guide, 2015.

[53] R. Hooke, T. A. Jeeves, Direct search solution of numerical and statistical problems, Journal of the ACM 8 (1961) 212-229. doi:10.1145/321062. 321069 .

[54] S. Kirkpatrick, Optimization by simulated annealing: Quantitative studies, Journal of Statistical Physics 34 (1984) 975-986. doi:10.1007/ BF01009452.

[55] B. Saavedra-Moreno, S. Salcedo-Sanz, A. Paniagua-Tineo, L. Prieto, A. Portilla-Figueras, Seeding evolutionary algorithms with heuristics for optimal wind turbines positioning in wind farms, Renewable Energy 36 (2011) 2838-2844. doi:/10.1016/j.renene.2011.04.018. 